\title{
Laparoscopic management of isolated traumatic gall bladder injury accompanied by operative cholangiogram (Case Study)
}

\author{
Abd Elrahman Elmaraghy, MD; Ahmed M Kmal, MD; \\ Rabbah Abd Ellateef, MSc
}

\author{
Department of General Surgery, Ain Shams University, Cairo, Egypt.
}

\begin{abstract}
Background and objectives: Blunt trauma to the gallbladder is a rare entity, particularly when no other organ is injured. In isolated blunt traumatic injury to the gallbladder, treatment options vary depending on the specific injury. The types of blunt trauma injuries to the gallbladder and their appropriate management are discussed. In addition, a case of blunt traumatic gall bladder injury that has been successfully managed with minimally invasive techniques is presented. This is accompanied by laparoscopic cholangiogram in order to precisely define extra hepatic biliary tree for possibly distorted anatomy and for exclusion of other biliary injury.

Methods: A patient with blunt abdominal trauma was safely managed with laparoscopic surgery for isolated gallbladder trauma. The preoperative and operative management are discussed as well as the application of minimally invasive surgery for this rare process.

Results: Laparoscopic cholecystectomy with intra-operative cholangiography was performed successfully. The patient did well postoperatively with no complications. No other injuries were identified at the time of laparoscopy.

Conclusion: Minimally invasive techniques may be safely applied to blunt trauma of the gallbladder in certain circumstances.
\end{abstract}

\section{Introduction:}

Gallbladder injury from blunt abdominal trauma is a rare event usually occurring in association with other intra-abdominal injuries. Isolated gallbladder injury is even more uncommon. ${ }^{1}$ Delayed presentation or deferred diagnosis of gallbladder injury is not uncommon due to the absence of related symptoms or vague complaints which may present days to weeks after the traumatic insult. ${ }^{2}$ Early diagnosis and treatment is essential to avoid serious morbidity and mortality. We present a patient with isolated gallbladder injury secondary to blunt abdominal trauma who was definitively managed laparoscopically.

\section{Case report:}

A 44-year-old female was the restrained front-seat passenger in a high-speed motor car accident. On arrival to our emergency room, she was awake and alert with a Glasgow Coma Score of 15 and complained of right upper quadrant (RUQ) abdominal pain. She was hemodynamically stable. A physical examination of her abdomen revealed a soft, non-distended abdomen with moderate RUQ tenderness but no palpable mass or signs of peritoneal irritation. Intestinal sound was normal. Plain radiographs of the cervical spine, chest, and pelvi-abdomen showed no evidence of acute injury. Laboratory findings were within normal limits, with the exception of hemoglobin of $10.0 \mathrm{gm} / \mathrm{dL}$. Computerized tomography of the abdomen showed a massively dilated gallbladder with pericholecystic fluid and heterogeneous intraluminal fluid consistent with blood and thrombus Figure(1). While under observation, the patient developed increasing RUQ pain and tenderness. A laparoscopic exploration was undertaken. Open technique was used for abdominal insufflation followed by routine $10 \mathrm{~mm}$ umbilical port. Laparoscopy revealed a massively dilated gallbladder without evidence of other injury Figure(2). Routine laparoscopic 
cholecystectomy was done starting by dissection of cystic duct and artery. An intraoperative cholangiogram was then conducted; an opening is made in the cystic duct using microscissors followed by selective cannulation using a 5-French straight Teflon catheter coaxially with 0.035 inch floppy tip guidewire. The guidewire is advanced 1-2 mm through the tip of the catheter and both are advanced to the common bile duct. Contrast injected \& a C-arm is used for fluoroscopy \& image acquisition Figure(3). No evidence of intra- or extrahepatic bile duct injury was present. Gross inspection of the specimen showed a markedly enlarged gallbladder filled with thrombus without evidence of cholelithiasis Figure(4). The patient made an uneventful recovery and was discharged home on the second postoperative day.

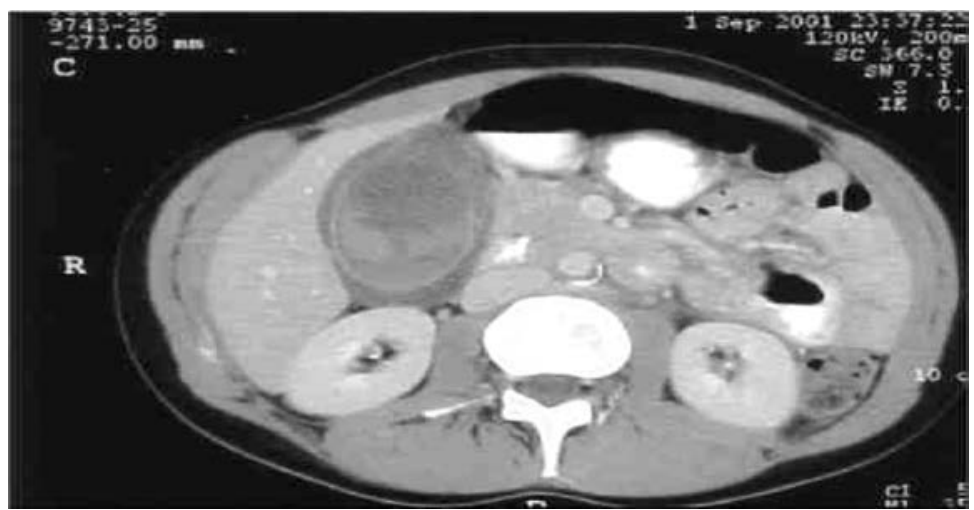

Figure (1): Preoperative CT scan showing markedly enlarged gallbladder with intraluminal blood and thrombus.

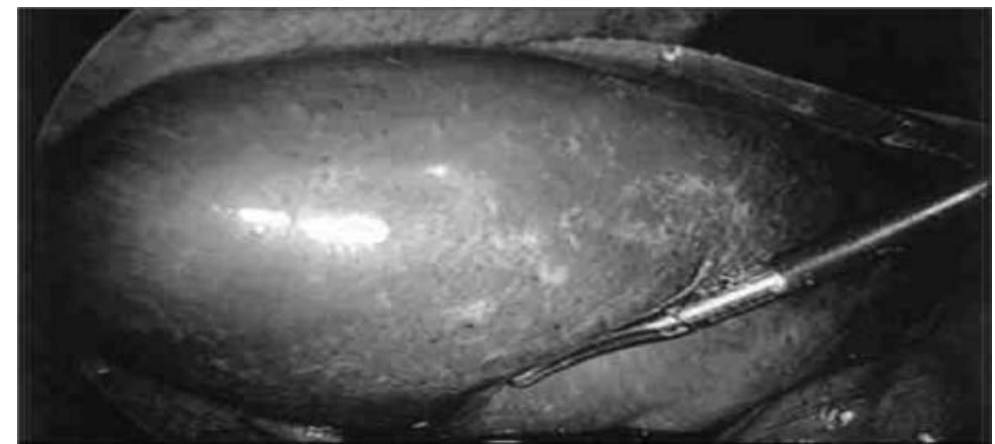

Figure (2): Intraoperative photograph illustrating markedly enlarged gallbladder.

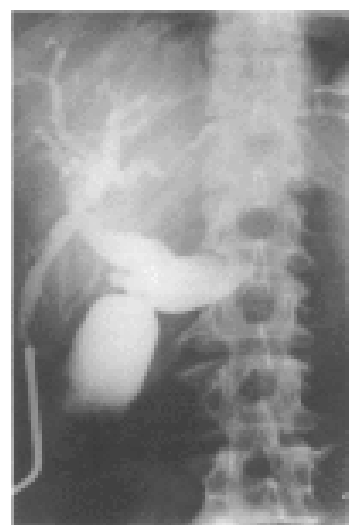

Figure (3): Intra-operative laparoscopic cholangiogram. 


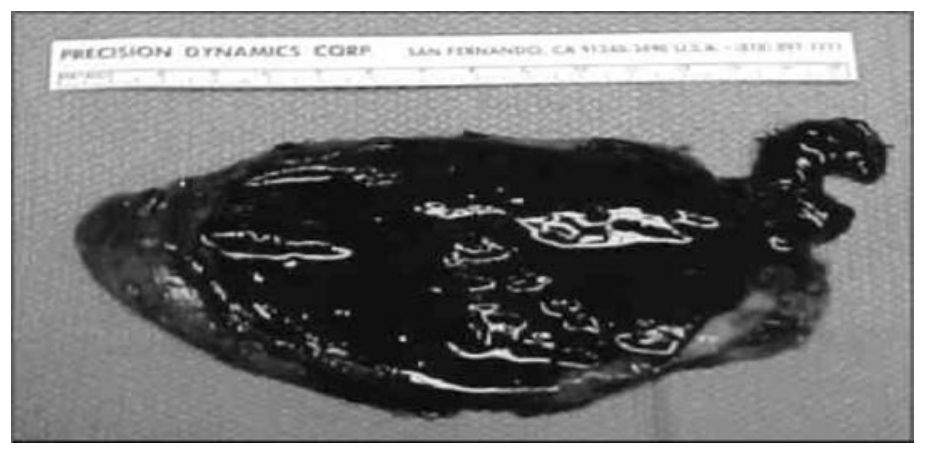

Figure (4): Gross photograph of the gallbladder specimen showing intraluminal thrombus.

\section{Discussion:}

Gallbladder injury occurs in less than $2 \%$ of blunt abdominal trauma cases and is usually associated with additional surrounding visceral injury. Isolated gallbladder injury is even rarer due to its position, protected by the liver, intestines, omentum, and the rib cage. Gallbladder injury is most commonly due to motor vehicle accidents, but may also result from a fall, blows to the abdomen, or crushing injury. ${ }^{2}$ Predisposing factors for gallbladder injury include alcohol consumption, which stimulates gastrin and secretin release, leading to an increase in bile flow and a subsequent pressure increase in the sphincter of Oddi; postprandial gallbladder distention; a thin-walled gallbladder; and malposition of the gallbladder. ${ }^{3}$

Early diagnosis of blunt gallbladder injury is crucial to lower the associated morbidity and mortality. However, delay is common due to vague or absent symptoms. ${ }^{4}$ Injury of the gallbladder typically presents in one of three ways. In one scenario, it is discovered during the operative evaluation and treatment of other intra-abdominal injuries. More commonly, gallbladder injury has an insidious onset multiple days after the traumatic event. ${ }^{5}$ Patients may return to the hospital after discharge with signs or symptoms of peritonitis or jaundice. The third group of individuals may present weeks later complaining of vague abdominal complaints. ${ }^{4}$

Injury to the gallbladder can be divided into three main classifications: (1) Laceration or perforation, which is the most common, usually affecting the fundus, (2) Avulsion of various degrees, ranging from partial-to-complete tear from the liver bed to traumatic cholecystectomy and disruption of the cystic duct and artery, and (3) Traumatic contusion, which is the least common, with an intramural hematoma developing that contributes to wall necrosis and subsequent perforation days to weeks after the initial traumatic insult. 6

Evaluation of the gallbladder in a patient with suspected injury may involve multiple modalities. Ultrasound will typically reveal a thickened hypoechoic edematous gallbladder wall, an echogenic pericholecystic fluid collection, a disruption of the gallbladder wall, and collapse of the lumen despite prolonged fasting. Computed tomography is a sensitive tool that may demonstrate gallbladder collapse, pericholecystic fluid collection, hyperdense intraluminal and extraluminal density, irregular gallbladder contour, and possible mass effect on the duodenum along with streaky mesenteric fat and omentum. Endoscopic retrograde cholangiopancreatography provides cursory information regarding injury and involvement of the liver parenchyma and ductal rupture, but may not completely identify the injury. Diagnostic peritoneal lavage rarely reveals bile in the lavage fluid although its presence may not signify biliary rupture. ${ }^{7}$

Treatment options vary from simple observation to cholecystectomy depending on the severity of injury. Most often, cholecystectomy is undertaken. Intraoperative evaluation of the biliary tree is imperative to rule out concomitant bile duct injury. We have demonstrated that laparoscopic surgical techniques may be safely used in instances where the likelihood of associated injuries is low, and definitive treatment can be rendered without increasing patient morbidity and mortality. When the injury is several days into 
its evolution or in the presence of inflammation a lateral approach (modified left lateral with right side up 60 degree, starting with $1 \mathrm{~cm}$ small incision at tip of eleventh costal cartilage) is most effective during dissection of the gallbladder to prevent injury to vital structures. Laparoscopic cholecystectomy for isolated gallbladder rupture in the case of blunt abdominal trauma may be an excellent option for many patients.

\section{References:}

1- Losanoff JE, Kjossev KT: Complete traumatic avulsion of the gallbladder. Injury 1999; 30: 365-368.

2- Sharma O: Blunt gallbladder injuries: Presentation of twenty-two cases with review of the literature. J Trauma 1995; 39: 576-580.
3- Chen X, Talner LB, Jurkovich GJ: Gallbladder avulsion due to blunt trauma. Am J Roentgenol 2003; 177: 822. 4- Smith SW, Hastings TN: Traumatic rupture of the gallbladder. Ann Surg 1954; 139 : 517-520.

5- Solheim K: Blunt gallbladder injury. Injury 1972; 3: 246-248.

6- Kohler R, Millin R, Bonner B, Louw A: Laparoscopic treatment of an isolated gallbladder rupture following blunt abdominal trauma in a schoolboy rugby player. Br J Sports Med 2002; 36: 378379.

7- Timothy R, Thoms L: Laparoscopic management of traumatic haemorrhagic cholecystitis. Journal of Society of Laparoscopic Surgery 2004; 8: 93-95. 